\title{
An Interaction Model for Long Pull-Down Menus
}

\author{
Hong-In Cheng $^{1}$ and Patrick E. Patterson ${ }^{*}, 2$ \\ ${ }^{1}$ Graduate School of Digital Design, Kyungsung University, Korea \\ ${ }^{2}$ Industrial Engineering, Texas Tech University, USA
}

\begin{abstract}
Previous studies have employed various models for exploring menu use, focusing mainly on short menus. In this study, long pull-down menus were examined in an item selection experiment. Our base model assumed that 1) the first eye fixation was located at the top, middle, or end of a menu depending on the search item; 2) menu scanning is either downward or upward; 3) the mouse was slowly moved downward from the top while scanning the target; 4) the user moves the mouse quickly to targets located at the edge of a menu; and 5) three items are scanned simultaneously. Additional models, developed by applying various assumptions, were evaluated. Experimental data showed that selection time increased based on item position and decreased for lower-positioned items in a menu having 28 alternatives, with more time needed for a menu having 50 choices because of scrolling.
\end{abstract}

\section{INTRODUCTION}

The pull-down menu is a popular interaction method used in modern user interfaces. To activate a pull-down menu, a user clicks on the menu title, searches for a target menu item, makes a decision, and then selects the needed choice. Thus, menu selection includes not only perceptive and cognitive elements, but also motor behavior.

When searching for alternatives, users display two distinct eye events:

1. The eyes may remain stationary (a fixation) for a short time, or

2. Movement occurs between two fixation points (a saccade) the trajectory of which is the scan path.

Two well-known models help describe menu selection (Fig. 1). The serial model assumes that a user perceives an item, recognizes the target, and then clicks on an item in serial order. The parallel model assumes a user moves his gaze and checks the items in working memory simultaneously, choosing targets as they are detected.

Norman [1] reported that menu selection is a serial process of search, choice, and response. He proposed that visual matching requires searching for and selecting a specific target, with the degree of similarity or ambiguity among alternative targets influencing search time. Because users need to read, understand, and assess each item, encoding and evaluation processes are used for partially specified targets. Norman presented three search models (Fig. 2).

When using a "serial inspection" method, users examine each item from the top on downward, without skipping items. Menu items are viewed without use of a pattern or repeated scanning in a "random search without replacement" method. In the "random search with replacement" method, items may be scanned repeatedly.

*Address correspondence to this author at the Industrial Engineering, Texas Tech University, USA; E-mail: Pat.Patterson@ttu.edu (a) Serial processing model

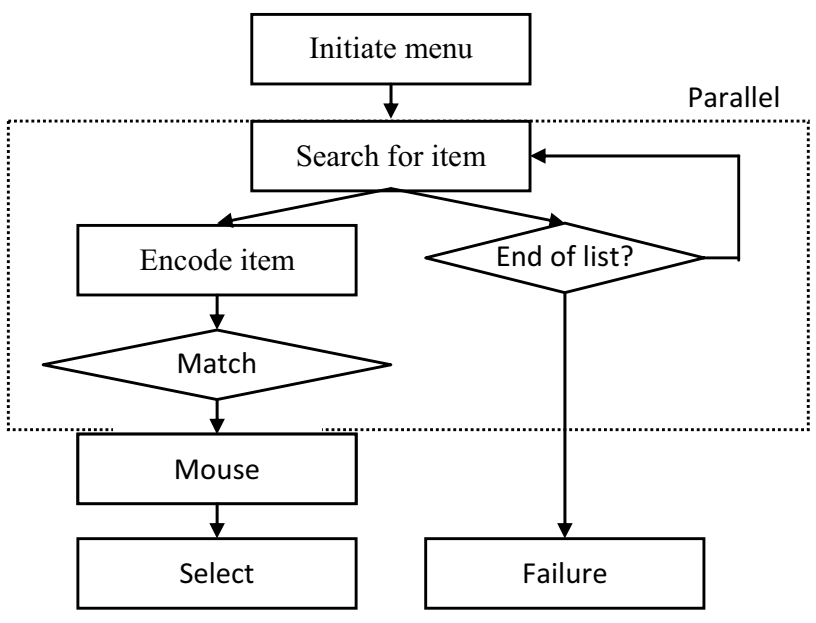

(b) Parallel processing model

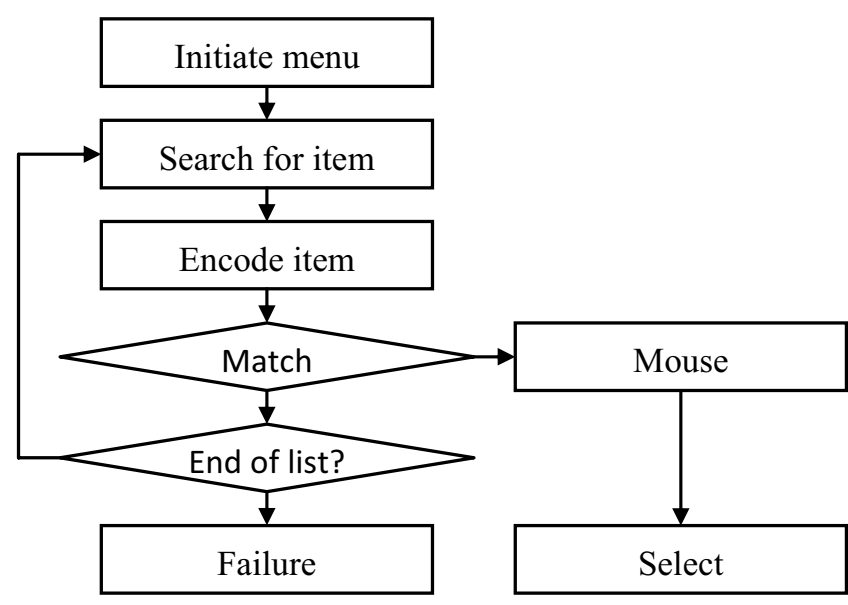

Fig. (1). Menu selection model. 
(a) Serial inspection

(b) Random without replacement
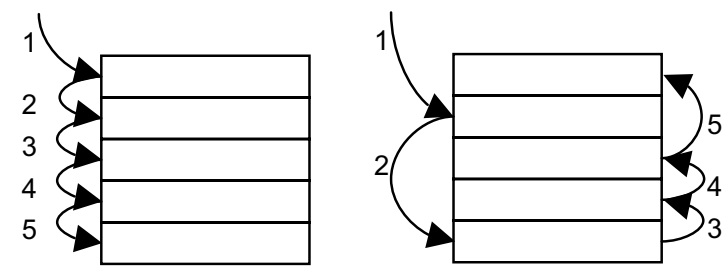

(c) Random with replacement

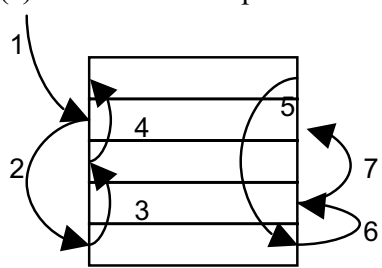

Fig. (2). Three models of visual search (Norman 1991).

Search strategies may also be classified according to the stopping rule used. An exhaustive search requires examining each item until the target is detected. Menu items are viewed again if the target is not found after all items have been observed. In a self-terminating search, users stop searching when the target is encountered. A self-terminating search is typically employed when users know exactly the target they are seeking.

The menu selection process has been compared to that used in choice reaction. In choice reaction, users typically select a response corresponding to the stimulus in a manner similar to menu selection, which requires item scanning and selection. Dutch physiologist Donders proposed that choice reaction time could be calculated by adding the times for simple reaction, stimulus classification, and response selection (as found in [2]).

Hick [3] and Hyman [4] suggested that choice reaction time is a function of the number of alternatives, for which the following quantitative model has been formulated:

$C T=\log _{2}(N+1)$,

where $C T$ is choice time, and $N$ is the number of alternatives.

Motor response time has been related to target distance and the difficulty of selecting the target (1). For an analog pointing device, Fitts' law predicts movement time thus:

$M T=a+b \log _{2}(A / W)$,

where $M T$ is movement time, $a$ and $b$ are constants, $A$ is distance to the target, $W$ is width of the target, and the logarithmic element is the index of difficulty. Two variations of Fitts' law have been suggested by Welford [5] and MacKenzie [6]:

$$
\begin{aligned}
& M T=a+b \log _{2}(A / W+0.5) \\
& M T=a+b \log _{2}(A / W+1)
\end{aligned}
$$

Equation 2", the Shannon formula, is preferred, as it fits data slightly better and has an index of difficulty that is always positive [7].
Lee and MacGregor [8] reported that selection time depends on search strategy, reading speed, and key press time. Their selection time was formulated thus:

$$
S=(E(A) \times t)+k+c
$$

where $\mathrm{S}$ is selection time, $\mathrm{E}(\mathrm{A})$ is the expected number of alternatives, $t$ is the time to read a single option, $k$ is key press time, and $c$ is computer response time. It was calculated that using between four and eight alternatives per page would minimize search time in a computerized information retrieval system.

The "Epic" model [9] proposes that 1) users employ both sequential and random searching strategies for scanning menu items, 2) multiple items are searched in parallel, 3) the travel distances of eye saccades are constant, and 4) mouse movement occurs after a target is found. The "ACT-R" model [10] predicts that 1) top-down search is used, 2) only single items are examined, 3 ) the travel distances of saccades vary, and 4) mouse movements follow the saccades before the discovery of chosen menu items.

Nielsen [11] performed an experiment using menus having three, six, and nine choices. Subjects selected single-digit menu items, randomly arranged, for each trial. The results showed that a linear relationship exists between the positions of menu items and selection time. The data also suggested that Fitts' law does not adequately explain menu selection time (although Nielsen's menu was not practical, since menu items were single-digit and randomly arranged for each selection).

Aaltonen et al. [12] reported that users scanned menu items in sequential sweeps by analyzing the scan path. However, because grouped menu items were used and sorted in random order within the groups, users in this study could not anticipate the locations of menu items.

Byrne et al. [13] used Nielsen's menu with six, nine, and twelve items, and proposed that 1) the primary search strategy is top-down, 2) some items are skipped during the topdown search, 3) these skipped items are occasionally found by backtracking, and 4) initial eye fixation is on one of the first three menu items. It was suggested that a more suitable model might exist between the explanations of the EPIC and the ACT-R models, and offered top-down search with occasional backtracking as a plausible model for short menus, with a top-to-bottom model for longer menus [13].

Hinckley et al. [14] distinguished two features of mouse movement, namely that users move the mouse slowly when distances are small, but more rapidly if distances are longer. Their experimental results also suggest that Fitts' law applies to scrolling times.

Previous studies $[9,13,15]$ used short menus and menu items that were not useful in practical design. For example, alternative menu items included digits and the order of menu items was random. More recently, long menus often have been used for e-commerce and other applications [16]. These longer menus require users to scroll up and down to search and select targets. It is not known how users interact with pull-down menus that require scrolling. This study examined 
search strategy and mouse movement using relatively long alphanumerically ordered menus and selected search strategy assumptions from previous studies to better model this interaction.

\section{METHOD}

\section{Subjects}

Fifty-two subjects ( 33 male, 19 female) from an introductory ergonomics class at Iowa State University received extra class credit for participating in the study. The subjects ranged from ages 19 to 39 (mean=21.2, $\mathrm{SD}=3.16$ ). Ninetysix percent of subjects indicated that they were familiar with the use of pull-down menus through their exposure to computer software.

\section{Interface}

Two different applications were designed for each menu. The subjects' first task was to select one of 28 department undergraduate courses and, in the second task, one of the 50 USA states. Users did not need to use any scrolling with the course menu. However, because not all the menu items fit onto a single screen, scrolling or clicking down through menu items was necessary to select a state (Fig. 3).

(a) 28 courses

Courses
IE 101 Orientation
IE 248 Manufacturing Process
IE 271 Applied Ergonomics
IE 298 Cooperative Education
IE 305 Engineering Economics
IE 312 Optimization
IE 318 Stochastic Analysis
IE 341 Production Systems
IE 348 Solidification Processes
IE 361 Quality Control
IE 375 Production Systems
IE 396 Summer Internship
IE 397 Engineering Internship
IE 398 Cooperative Education
IE 408 Problem Solving
IE 409 Systems Effectiveness
IE 419 Manufact. Sys. Modeling
IE 436 Reliability Engineering
IE 439 Industrial Automation
IE 441 Industrial Eng. Design
IE 448 manufact. Sys.Eng.
IE 449 CAD and Manufacturing
IE 465 Knowledge Engineering
IE 466 Multi.Eng.Design
IE 471 Safety and Reliability
IE 481 E-Commerce Engineering
IE 490 Independent Study
IE 498 Coopertive Education

Fig. (3). Screen shot of pull-down menus.

\section{Procedures}

Purpose, procedure, risks, and benefits of the experiments were explained to subjects and informed consent was obtained. The participants then selected an experimental sheet from a stack of papers and filled out personal information before the experiment. The experimental sheet was composed of three sections, 1) name, age, and gender; 2) three randomly pre-selected items for each menu; and 3) record of the response time. Response time was measured in milliseconds by a JAVA applet.

Each subject was presented with the two menus and given a short time to examine each menu, as well as the order of items for both menus. The subjects also chose four randomly selected trial items for each menu. When the subjects felt comfortable with the menus and the environment, actual selection options were given them from the selected experimental sheet. The subjects had to select specific menu items twice within a randomly presented menu order. Menus were programmed always to appear in the same position on the screen.

\section{RESULTS}

\section{Course Selection Task}

The mean times for menu item selection for the courses (Fig. 4) show a gradual increase in search time, depending on item position, with times decreasing toward the end of the menu. We developed a basic model, and then changed each assumption to develop a model agreeing more closely to our experimental data. Our first model presumed that 1) the first eye fixation is directed to the top, middle, or end of the menu depending on search item characteristics; 2) menu scanning is either downward or upward; 3) the user moves the mouse quickly to targets located at the edges; and 4) three items are scanned simultaneously. First eye fixations were assumed to be located at the top, middle, or end of the menu as the menus were long and items were in either alphabetic or numeric order. Based on these assumptions, an equation was obtained using linear regression (Equation 4).

$$
\begin{aligned}
S T & =217+13.489 \log _{2}(N+1) \\
& +479.455 \log _{2}\left(\frac{0.9 \times A}{W}+1\right), \\
r^{2} & =0.83
\end{aligned}
$$

where $\mathrm{N}$ is the number of alternatives to scan, $\mathrm{A}$ the distance to the target, and $\mathrm{W}$ the smaller of height or width. The last five items were assumed to be closer because of rapid mouse movement towards targets at the edge. Edge targets represent items located near the borders of the menu. Because users can skip to the lower boundary without encoding intervening menu items, edge targets induce rapid mouse movement.

A second model ignoring the quick edge movement used in the model above did not represent the user behavior well $\left(\mathrm{r}^{2}=0.705\right.$, Fig. 5). Results were also unsatisfactory when search and movement were assumed to occur serially $\left(r^{2}=0.706\right.$, Fig. 5).

A third model was developed which assumed that 1) first fixation stays on the first menu item, 2) a top-down search 


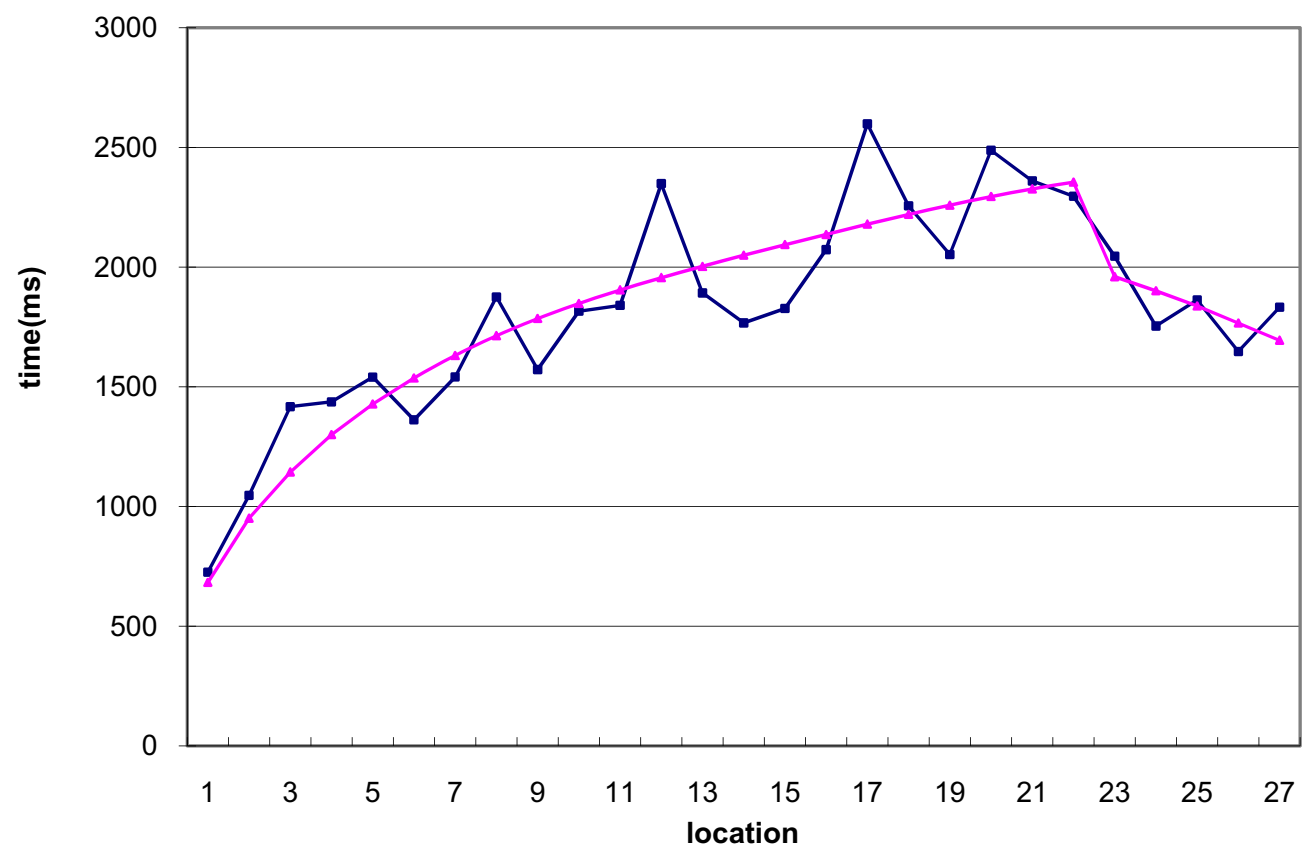

Fig. (4). The selection time of the courses based on the location of menu-items (Square: experimental data, Triangle: predicted time by equation 4).

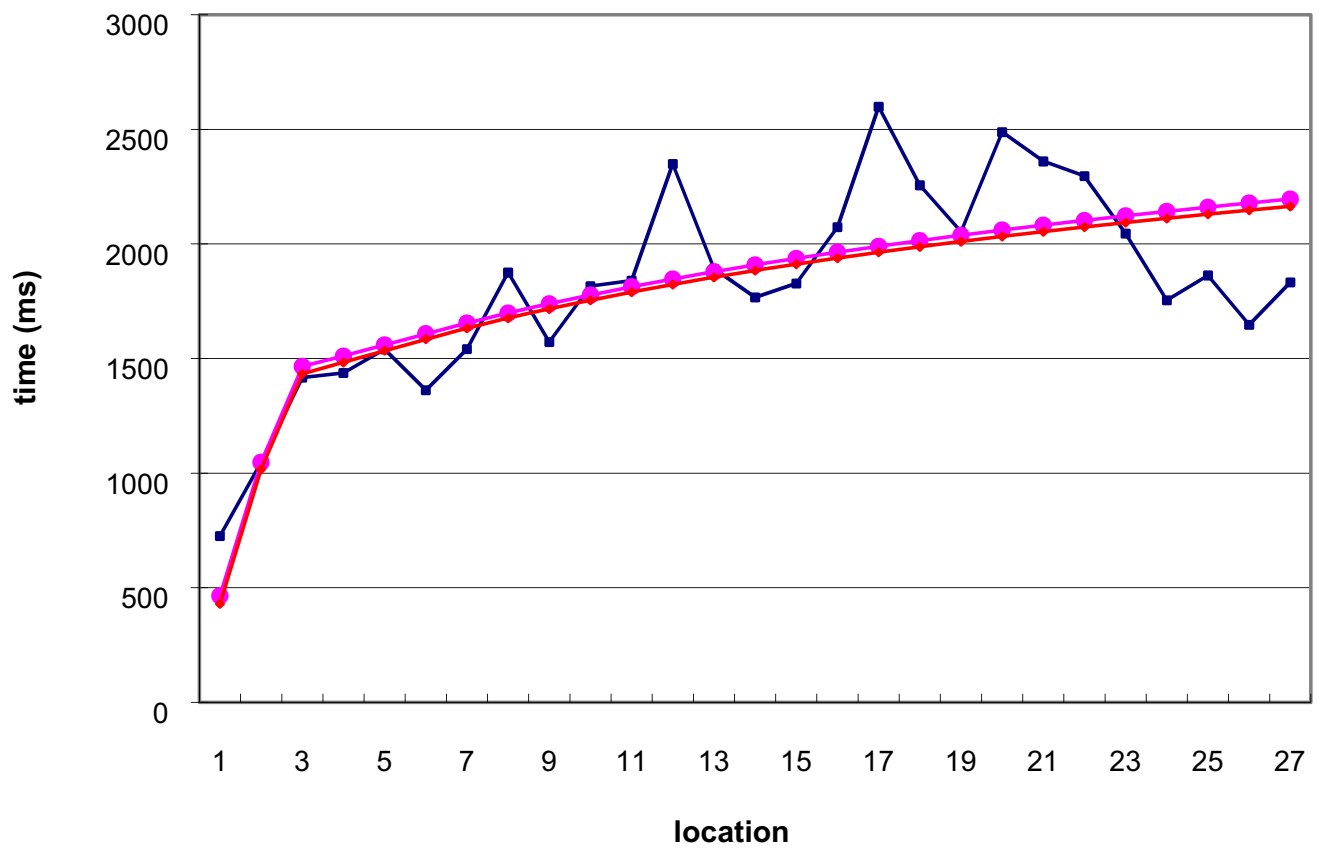

Fig. (5). The selection time of the courses (Square: experimental data, Circle: no edge movement, Diamond: serial search).

strategy is utilized, 3) the mouse moves slightly from the top downwards while searching the target, 4) the user moves the mouse quickly to an edge target, and 5) three items are scanned simultaneously. Selection time was formulated in the following manner:

$$
\begin{aligned}
S T & =247.582-38.78 \log _{2}(N+1) \\
& +521.785 \log _{2}\left(\frac{0.9 \times A}{W}+1\right), \\
r^{2} & =0.831
\end{aligned}
$$

\section{State Selection Task}

Because every item could not be displayed on a screen, the pull-down menu of 50 states required scrolling to select an item located in a lower position. About thirty alternatives could be viewed without scrolling (Fig. 3). The data show a steady increase in time up to the thirtieth item, a rapid increase in time between the thirty-first and forty-third items, and decreased times for items toward the end of the menu (Fig. 6).

Our model for selecting among 28 Industrial Engineering courses was adapted to the 50 -state menu by assuming that 1) the initial fixation stays at either the top or bottom of the screen; 2) the menu search is either downward or upward; 3) 


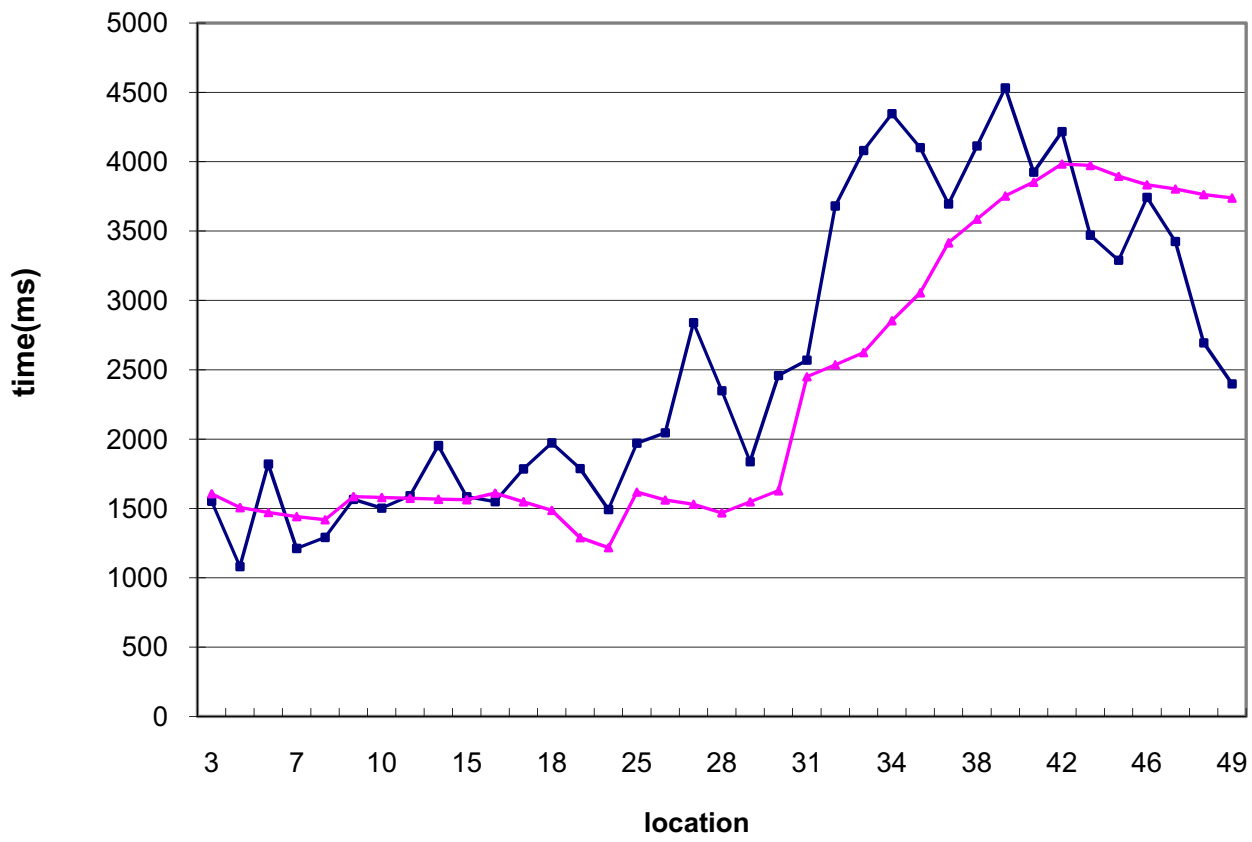

Fig. (6). Selection times for 50 states (Square: experimental data, Triangle: expected time).

the mouse moves slowly downward while the target is scanned; 4) the user moves the mouse to the bottom rapidly, without pausing for an edge target; 5) three items are scanned simultaneously; and 6) scrolling time is explained by Fitts' law [14]. Using linear regression, the model was formulated as $\left(\mathrm{r}^{2}=0.891\right)$ :

$$
\begin{aligned}
S T & =642.692+221.511 \log _{2}(N+1) \\
& +216.84 \log _{2}\left(\frac{0.9 \times A}{W}+1\right) \\
& +656.007 \log _{2}\left(\frac{S}{W}\right)
\end{aligned}
$$

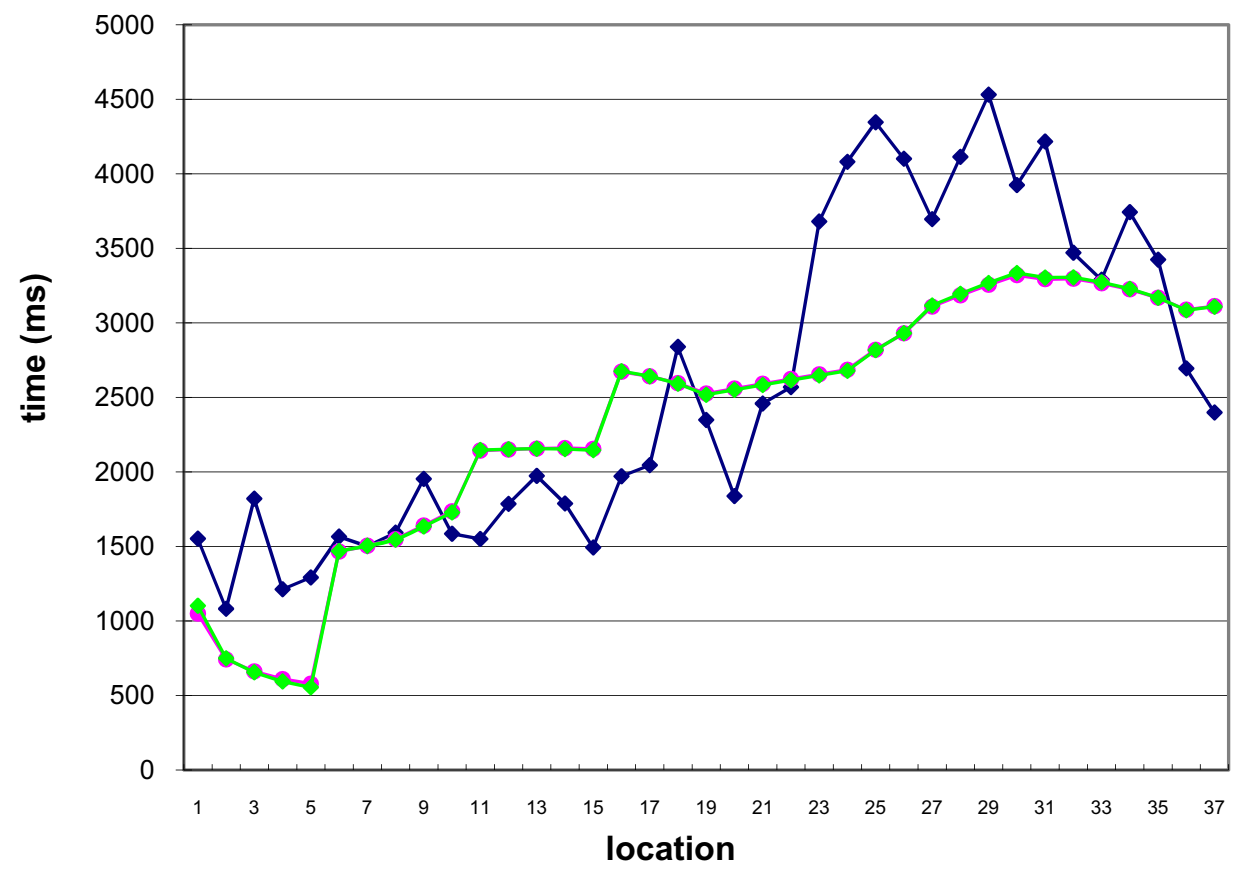

Fig. (7). Selection time among 50 states (Square: experimental data, Circle: no edge movement, Diamond: serial search). where $\mathrm{N}$ is the number of menu items to be scanned, $\mathrm{A}$ is distance to the target, $\mathrm{W}$ is the smaller of height or width, and $\mathrm{S}$ is scrolling distance. The data could not be explained well when the quick edge movement assumption was ignored $\left(r^{2}=0.671\right.$, Fig. 7), nor without serial mouse movement $\left(r^{2}=0.672\right.$, Fig. 7). Since the upper 30 items may be viewed without scrolling, we did not assume scrolling (however, users occasionally still scrolled to search or select among items). The probabilities of scrolling use can be found in Table $\mathbf{1}$.

A second model was developed by assuming that 1) initial fixation is directed at the first menu item, 2) the search 
strategy is top-down, 3) the mouse is moved one-percent of selection distance during scanning, 4) the mouse is moved rapidly to the edge target, and 5) three items are scanned simultaneously. The mathematical model using these assumptions was $\left(\mathrm{r}^{2}=0.884\right)$ :

$$
\begin{aligned}
S T & =1955.806-281.001 \log _{2}(N) \\
& -177.235 \log _{2}\left(\frac{0.9 \times A}{W}+1\right)+773.056 \log _{2}\left(\frac{S}{W}\right)
\end{aligned}
$$

When the edge benefit and preceding mouse movement were ignored, experimental data were not adequately explained.

Table 1. Probability of Scrolling (0 is Top of Menu, 50 is Bottom)

\begin{tabular}{|c|c|}
\hline Location of Item & Probability \\
\hline \hline $0 \sim 10$ & 0.1 \\
\hline $11 \sim 15$ & 0.2 \\
\hline $16 \sim 20$ & 0.4 \\
\hline $21 \sim 30$ & 0.6 \\
\hline $31 \sim 50$ & 1 \\
\hline
\end{tabular}

\section{DISCUSSION}

Typically, models have been developed for interacting with menu items by examining short pull-down menus. In this study, longer pull-down menus were designed and studied to examine such interactions. The first menu, containing 28 university courses, did not require scrolling; however, scrolling was necessary to access lower items on the 50 -state menu.

Selection time for the course menu exhibited two primary characteristics: a proportional time increase related to menu position and decreased selection time relative to items lower in the menu (Fig. 5). Because users could guess the rough locations of menu items in advance when alternatives were arranged alphanumerically and the menus were long, we considered the initial eye fixation to be directed at the top, middle, or end of the menu depending on the search item. For example, if users must select "'Wyoming"" from the states menu they didn't start scanning the items from the top of the menu, knowing the items were in alphabetical order. Scanning was then directed either upward or downward from this point (a downward search would be employed when the target is located below the point of fixation).

Unconscious movements of the cursor following saccade were observed as predicted and selection time was reduced by using this preliminary motion. When a user recognized the target was close to the end of a menu, the cursor moved to the final approximate location and the target was chosen quickly. Scrolling was predicted by Fitts' law. Except for adding scrolling and the position of the first fixation, it was shown the selection process for longer menus was similar to that of shorter menus.
An abrupt increase in selection time after the 30th item in the 50-state menu resulted because of the need for scrolling to locate items lower in the menu. Also, because it was difficult to guess where a given item might be located in the longer list, we discovered that initial fixation was not directed toward the middle of that portion of the menu visible on the screen. The first fixation was therefore assumed to be either at the top or the bottom of the menu displayed. However, when considering the entire menu, the first fixation did move toward the top, middle, or bottom items in a manner similar to that found for the course menu.

Users often moved the cursor to the lower items even if scrolling was not needed to locate a targeted item. To explain this, stochastic scrolling was introduced to the model. As Hinckley et al. [14] reported, Fitts' law predicted scrolling time well, and our model became more accurate as menu length increased. However, other approaches did not show similar results.

A top-down serial scan model also offered good results. Nielsen's menu showed that initial fixation was frequently located at the first item [13]. However, because menu items were rearranged randomly in each trial, users could not predict their locations within the menu. Provided the menu is reasonably well arranged, we feel that our model is more likely.

Three possible menu improvements may be directly induced from the study: more efficient first fixation, better scrolling, and the use of quicker edge movement. Menu designers need to consider how users might better position their first fixations. As used recently, an indexed menu is a good possibility for improving initial eye fixation. New scrolling methods (for example, automatic scrolling) might also be usefully studied. Edge target approaches can be widely adopted, especially for emergency buttons.

\section{REFERENCES}

[1] Norman KL. The psychology of menu selection: Designing cognitive control at the human-computer interface. Westport CT: Greenwood Publishing Group, Inc.; 1991.

[2] Kantowitz BH, Sorkin RD. Human factors. New York: Wiley; 1983.

[3] Hick WE. On the rate of gain of information. J Exp Psychol 1952; p. 11-26.

[4] Hyman R. Stimulus information as a determinant of reaction time. J Exp Psychol 1953; 45: 188-99.

[5] Welford AT. Fundamentals of skill. London: Methen; 1968

[6] MacKenzie IS. A note on the information-theoretic basis for Fitts' law. J Mot Behav 1989; 21: 323-30.

[7] MacKenzie IS. Fitts' law as a research and design tool in humancomputer interaction. Int J Hum Comput Interact. 1992; 7: 91-139.

[8] Lee E, MacGregor J. Minimizing user search time in menu retrieval systems. Hum Factors 1985; 27(2): 157-62.

[9] Hornof AJ, Kieras DE. Cognitive modeling reveals menu search is both random and systematic. Human Factors in Computing Systems, 1997: Proceedings of CHI 97; 1997. p. 107-14.

[10] Anderson JR, Matessa M, Lebiere C. ACT-R: A theory of higher level cognition and its relation to visual attention. Hum Comput Interact 1997; 12(4): 439-62.

[11] Nilsen, EL. Perceptual-motor control in human computer interaction (Tech. Rep. No. 37). Ann Arbor, Michigan: The Cognitive Science and Machine Intelligence Laboratory, The University of Michigan; 1991. 
[12] Aaltonen A, Hyrskykari A, Räihä K-J. 101 spots, or how do users read menus? Human factors in computing systems: Proceedings of CHI 98; 1998. p. 132-39.

[13] Byrne MD, Anderson JR, Douglass S, Matessa M. Eye tracking the visual search of click-down menu. Human factors in computing systems: Proceedings of CHI 99; 1999. p. 402-9.

[14] Hinckley K, Cutrell E, Bathiche E, Muss T. Quantitative analysis of scrolling techniques. Human factors in computing systems: Proceedings of CHI 2002; 2002. p. 65-72.
[15] Hornof AJ, Kieras DE. Cognitive modeling demonstrates how people use anticipated location knowledge of menu items. Human Factors in Computing Systems: Proceedings of CHI 9; 1999. p. 410-17.

[16] Cheng H-I, Patterson PE. The grid menu: efficient and robust selection of menu-items. Proceedings of the Human Factors and Ergonomics Society 46th Annual Meeting; 2002. p. 1281-85.

(C) Cheng and Patterson; Licensee Bentham Open.

This is an open access article distributed under the terms of the Creative Commons Attribution License (http://creativecommons.org/license/by/2.5/), which permits unrestrictive use, distribution, and reproduction in any medium, provided the original work is properly cited 